Kragujevac Journal of Mathematics

Volume 40(2) (2016), Pages 224-236.

\title{
HIGH DIMENSIONAL FRACTIONAL COUPLED SYSTEMS: NEW EXISTENCE AND UNIQUENESS RESULTS
}

\author{
LOUIZA TABHARIT ${ }^{1}$ AND ZOUBIR DAHMANI ${ }^{2}$
}

\begin{abstract}
In this paper, we study a class of high dimensional coupled fractional differential systems using Caputo approach. We investigate the existence of solutions using Schaefer fixed point theorem. Moreover, new existence and uniqueness results are obtained by using the contraction mapping principle. Finally, Some examples are presented to illustrate our main results.
\end{abstract}

\section{Introduction AND PRELIMINARIES}

Fractional differential equations have gained a great interest because of their many applications in modeling of physical and chemical processes and in engineering sciences. For the basic theory of fractional differential equations, see $[1-10,12-14,17,22]$. Moreover, the nonlinear coupled systems involving fractional derivatives are also very important, since they occur in various problems of applied mathematics. In the literature, we can find many papers dealing with the existence and uniqueness of solutions. For more details, we refer the reader to [11,16,18-21] and the references therein.

Motivated by cited coupled systems-papers, in this work, we discuss the existence and uniqueness of solutions for the following problem:

Key words and phrases. Banach contraction principle, Caputo derivative, fixed point, differential equation, existence, uniqueness.

2010 Mathematics Subject Classification. 30C45, 39B72, 39B82.

Received: October 12, 2015.

Accepted: December 4, 2015. 


$$
\left\{\begin{array}{l}
D^{\alpha_{1}} u_{1}(t)=\sum_{i=1}^{l} f_{i}^{1}\left(t, u_{1}(t), \ldots, u_{m}(t), D^{\gamma_{1}} u_{1}(t), \ldots, D^{\gamma_{m}} u_{m}(t)\right), \quad t \in J, \\
D^{\alpha_{2}} u_{2}(t)=\sum_{i=1}^{l} f_{i}^{2}\left(t, u_{1}(t), \ldots, u_{m}(t), D^{\gamma_{1}} u_{1}(t), \ldots, D^{\gamma_{m}} u_{m}(t)\right), \quad t \in J, \\
\vdots \\
D^{\alpha_{m}} u_{m}(t)=\sum_{i=1}^{l} f_{i}^{m}\left(t, u_{1}(t), \ldots, u_{m}(t), D^{\gamma_{1}} u_{1}(t), \ldots, D^{\gamma_{m}} u_{m}(t)\right), \quad t \in J, \\
u_{k}(0) \quad=a_{0}^{k}, \\
u_{k}^{(j)}(0) \quad=0, \quad j=1,2, \ldots, n-2, \\
u_{k}^{(n-1)}(0)=J^{r_{k}} u_{k}\left(\tau_{k}\right), \quad r_{k}>0,
\end{array}\right.
$$

where $k=1,2, \ldots, m$. We suppose that $\left.n-1<\alpha_{k}<n, \gamma_{k} \in\right] 0, n-1[, k=$ $\left.1,2, \ldots, m, n \in \mathbb{N}^{*}-\{1\}, m, l \in \mathbb{N}^{*}, \tau \in\right] 0,1\left[, J:=[0,1]\right.$. The derivatives $D^{\alpha_{k}}, D^{\gamma_{k}}$, $k=1,2, \ldots, m$, are taken in the sense of Caputo. For the functions $\left(f_{i}^{k}\right)_{i=1, \ldots, l}^{k=1,2, \ldots}$ : $J \times \mathbb{R}^{2 m} \rightarrow \mathbb{R}$, we will specify them later.

The paper is organised as follows: We begin by introducing some definitions and lemmas that will be used in the proof of the main results. Then, in the Main Results Section, we prove the existence of solutions theorems. At the last section, some illustrative examples are treated. So, let us now present the basic definitions and lemmas [15].

Definition 1.1. The Riemann-Liouville fractional integral operator of order $\alpha>0$, for a continuous function $f$ on $[0, \infty[$ is defined as:

$$
J^{\alpha} f(t)= \begin{cases}\frac{1}{\Gamma(\alpha)} \int_{0}^{t}(t-s)^{\alpha-1} f(s) d s, & \alpha>0, t \geq 0, \\ f(t), & \alpha=0, t \geq 0,\end{cases}
$$

where $\Gamma(\alpha):=\int_{0}^{\infty} e^{-x} x^{\alpha-1} d x$.

Definition 1.2. The Caputo derivative of order $\alpha$ for a function $u:[0, \infty) \rightarrow \mathbb{R}$, which is at least $n$-times differentiable can be defined as:

$$
D^{\alpha} u(t)=\frac{1}{\Gamma(n-\alpha)} \int_{0}^{t}(t-s)^{n-\alpha-1} u^{(n)}(s) d s=J^{n-\alpha} u^{(n)}(t),
$$

for $n-1<\alpha<n, n \in \mathbb{N}^{*}-\{1\}$.

We recall the following lemmas $[7,19]$. 
Lemma 1.1. For $\alpha>0$, the general solution of the fractional differential equation $D^{\alpha} u(t)=0$, is given by

$$
u(t)=\sum_{j=0}^{n-1} c_{j} t^{j}
$$

where $c_{j} \in \mathbb{R}, j=0, \ldots, n-1, n=[\alpha]+1$.

Lemma 1.2. Let $\alpha>0$. Then

$$
J^{\alpha} D^{\alpha} u(t)=u(t)+\sum_{j=0}^{n-1} c_{j} t^{j}
$$

where $c_{j} \in \mathbb{R}, j=0,1, \ldots, n-1, n=[\alpha]+1$.

Lemma 1.3. Let $q>p>0, g \in L^{1}([a, b])$. Then $D^{p} J^{q} f(t)=J^{q-p} f(t), t \in[a, b]$.

Lemma 1.4 (Schaefer fixed point Theorem). Let $E$ be Banach space and $T: E \rightarrow E$ is a completely continuous operator. If $V=\{u \in E: u=\mu T u, 0<\mu<1\}$ is bounded, then $T$ has a fixed point in $E$.

The proof of the following auxiliary lemma is crucial for the problem (1.1).

Lemma 1.5. Assume that $\left(Q_{i}^{k}\right)_{i=1, \ldots, l}^{k=1, \ldots, m} \in C([0,1], \mathbb{R}), m, l \in \mathbb{N}^{*}$. And consider the problem

$$
D^{\alpha_{k}} u_{k}(t)=\sum_{i=1}^{l} Q_{i}^{k}(t), \quad t \in J, n-1<\alpha_{k}<n, n \in \mathbb{N}^{*}-\{1\},
$$

associated with the conditions:

$$
\begin{cases}u_{k}(0) & =a_{0}^{k}, \\ u_{k}^{(j)}(0) & =0, \quad j=1,2, \ldots, n-2, \\ u_{k}^{(n-1)}(0) & =J^{r_{k}} u_{k}\left(\tau_{k}\right),\end{cases}
$$

where $k=1,2, \ldots, m$. Then for all $k=1,2, \ldots, m$, we have

$$
\begin{aligned}
u_{k}(t)=-\sum_{i=1}^{l} \int_{0}^{t} \frac{(t-s)^{\alpha_{k}-1}}{\Gamma\left(\alpha_{k}\right)} Q_{i}^{k}(s) d s+a_{0}^{k}+\frac{\Gamma\left(r_{k}+n\right) t^{n-1}}{(n-1) !\left(\tau_{k}^{r_{k}+n-1}-\Gamma\left(r_{k}+n\right)\right)} \\
\quad \times\left(\sum_{i=1}^{l} \int_{0}^{\tau_{k}} \frac{\left(\tau_{k}-s\right)^{\alpha_{k}+r_{k}-1}}{\Gamma\left(\alpha_{k}+r_{k}\right)} Q_{i}^{k}(s) d s-\frac{a_{0}^{k} \tau_{k}^{r_{k}}}{\Gamma\left(r_{k}+1\right)}\right) .
\end{aligned}
$$

Proof. Thanks to (1.2) and by (1.3), we have

$$
u_{k}(t)=\sum_{i=1}^{l} \int_{0}^{t} \frac{(t-s)^{\alpha_{k}-1}}{\Gamma\left(\alpha_{k}\right)} Q_{i}^{k}(s) d s-c_{0}^{k}-c_{1}^{k} t-c_{2}^{k} t^{2}-\cdots-c_{n-1}^{k} t^{n-1},
$$


where $c_{j}^{k} \in \mathbb{R}, j=0,1,2, \ldots, n-1, k=1,2, \ldots, m, m \in \mathbb{N}^{*}$, and $n-1<\alpha_{k}<n$, $n \in \mathbb{N}^{*}-\{1\}$.

For all $k=1,2, \ldots, m$, we observe that

$$
\begin{cases}u_{k}(0) & =-c_{0}^{k}, \\ u_{k}^{(j)}(0) & =-j ! c_{j}^{k}, \quad j=1,2, \ldots, n-2, \\ u_{k}^{(n-1)}(0) & =-(n-1) ! c_{n-1}^{k} .\end{cases}
$$

The conditions (1.4) will allow us to obtain

$$
\left\{\begin{array}{l}
c_{0}^{k}=-a_{0}^{k}, \\
c_{j}^{k}=0, \quad j=1,2, \ldots, n-2, \\
c_{n-1}^{k}=\frac{\Gamma\left(r_{k}+n\right)}{\Gamma(n)\left(\tau_{k}^{r_{k}+n-1}-\Gamma\left(r_{k}+n\right)\right)}\left(a_{0}^{k} \frac{\tau_{k}^{r_{k}}}{\Gamma\left(r_{k}+1\right)}-\sum_{i=1}^{l} J^{\alpha_{k}+r_{k}} Q_{i}^{k}\left(\tau_{k}\right)\right)
\end{array}\right.
$$

where $k=1,2, \ldots, m$. The values of $c_{j}^{k}$ given by (1.7) replaced in (1.6) imply (1.5). This completes the proof of Lemma 1.5.

Now, we consider the space:

$$
S:=\left\{\left(u_{1}, u_{2}, \ldots, u_{n}\right): u_{k} \in C([0,1], \mathbb{R}), D^{\gamma_{k}} u_{k} \in C([0,1], \mathbb{R}), k=1,2, \ldots, m\right\},
$$

endowed with the norm

$$
\left\|\left(u_{1}, u_{2}, \ldots, u_{m}\right)\right\|_{S}=\max _{1 \leq k \leq m}\left(\left\|u_{k}\right\|_{\infty},\left\|D^{\gamma_{k}} u_{k}\right\|_{\infty}\right) .
$$

This Banach space will be used in the proof of the theorems.

\section{MAin Results}

We impose the following hypotheses:

$\left(H_{1}\right)$ : There exist nonnegative constants $\left(\mu_{i, i=1, \ldots, l}^{k, k, \ldots, 1,2, \ldots, m}\right)_{j, j=1,2, \ldots, 2 m}$, such that for all $t \in[0,1]$ and for all $\left(u_{1}, u_{2}, \ldots, u_{2 m}\right),\left(v_{1}, v_{2}, \ldots, v_{2 m}\right) \in \mathbb{R}^{2 m}$, we have

$$
\left|f_{i}^{k}\left(t, u_{1}, u_{2}, \ldots, u_{2 m}\right)-f_{i}^{k}\left(t, v_{1}, v_{2}, \ldots, v_{2 m}\right)\right| \leq \sum_{j=1}^{2 m}\left(\mu_{i}^{k}\right)_{j}\left|u_{j}-v_{j}\right| .
$$

$\left(H_{2}\right)$ : The functions $\left(f_{i}^{k}\right)_{i=1,2, \ldots, l}^{k=1,2, \ldots, m}:[0,1] \times \mathbb{R}^{2 m} \rightarrow \mathbb{R} ; m, l \in \mathbb{N}^{*}$ are continuous.

$\left(H_{3}\right)$ : There exist nonnegative functions $\left(\omega_{i}^{k}\right)_{i=1, \ldots, l}^{k=1, \ldots, m} \in C([0,1])$, such that: for all $t \in[0,1]$ and for all $\left(u_{1}, u_{2}, \ldots, u_{2 m}\right) \in \mathbb{R}^{2 m}$

$$
\left|f_{i}^{k}\left(t, u_{1}, u_{2}, \ldots, u_{2 m}\right)\right| \leq \omega_{i}^{k}(t)
$$

with

$$
\sup _{t \in J} \omega_{i}^{k}(t)=C_{i}^{k} .
$$


Setting the following quantities:

$$
\begin{aligned}
\Sigma_{k} & =\sum_{j=1}^{2 m} \sum_{i=1}^{l}\left(\mu_{i}^{k}\right)_{j}, \\
\digamma_{k} & =\frac{1}{\Gamma\left(\alpha_{k}+1\right)}+\frac{\Gamma\left(r_{k}+n\right) \tau_{k}^{\alpha_{k}+r_{k}}}{(n-1) !\left|\tau_{k}^{r_{k}+n-1}-\Gamma\left(r_{k}+n\right)\right| \Gamma\left(\alpha_{k}+r_{k}+1\right)}, \\
\digamma_{k}^{*} & :=\frac{1}{\Gamma\left(\alpha_{k}-\gamma_{k}+1\right)}+\frac{\Gamma\left(r_{k}+n\right) \tau_{k}^{\alpha_{k}+r_{k}}}{\Gamma\left(n-\gamma_{k}\right)\left|\tau_{k}^{r_{k}+n-1}-\Gamma\left(r_{k}+n\right)\right| \Gamma\left(\alpha_{k}+r_{k}+1\right)},
\end{aligned}
$$

and

$$
\begin{aligned}
W_{k} & =\left|a_{0}^{k}\right|+\frac{\Gamma\left(r_{k}+n\right)\left|a_{0}^{k}\right| \tau_{k}^{r_{k}}}{(n-1) !\left|\tau_{k}^{r_{k}+n-1}-\Gamma\left(r_{k}+n\right)\right| \Gamma\left(r_{k}+1\right)}, \\
W_{k}^{*} & =\frac{\Gamma\left(r_{k}+n\right)\left|a_{0}^{k}\right| \tau_{k}^{r_{k}}}{\Gamma\left(n-\gamma_{k}\right)\left|\tau_{k}^{r_{k}+n-1}-\Gamma\left(r_{k}+n\right)\right| \Gamma\left(r_{k}+1\right)} .
\end{aligned}
$$

We now prove the first main result.

Theorem 2.1. Assume that $\left(H_{1}\right)$ and the following are satisfied

$$
\max _{1 \leq k \leq m} \Sigma_{k}\left(\digamma_{k}, \digamma_{k}^{*}\right)<1 .
$$

Then, the problem (1.1) has a unique solution on $J$.

Proof. Let us take the operator $P: S \rightarrow S$ given by $P\left(u_{1}, u_{2}, \ldots, u_{m}\right)(t):=\left(P_{1}\left(u_{1}, u_{2}, \ldots, u_{m}\right)(t), \ldots, P_{m}\left(u_{1}, u_{2}, \ldots, u_{m}\right)(t)\right), \quad t \in J$, such that

$$
\begin{aligned}
& P_{k}\left(u_{1}, u_{2}, \ldots, u_{m}\right)(t) \\
& :=-\sum_{i=1}^{l} \int_{0}^{t} \frac{(t-s)^{\alpha_{k}-1}}{\Gamma\left(\alpha_{k}\right)} f_{i}^{k}\left(t, u_{1}(t), \ldots, u_{m}(s), D^{\gamma_{1}} u_{1}(s), \ldots, D^{\gamma_{m}} u_{m}(s)\right) d s+a_{0}^{k} \\
& +\frac{\Gamma\left(r_{k}+n\right) t^{n-1}}{(n-1) !\left(\tau_{k}^{r_{k}+n-1}-\Gamma\left(r_{k}+n\right)\right)} \\
& \quad \times\left(\sum_{i=1}^{l} \int_{0}^{\tau_{k}} \frac{\left(\tau_{k}-s\right)^{\alpha_{k}+r_{k}-1}}{\Gamma\left(\alpha_{k}+r_{k}\right)} f_{i}^{k}\left(t, u_{1}(t), \ldots, u_{m}(s), D^{\gamma_{1}} u_{1}(s), \ldots, D^{\gamma_{m}} u_{m}(s)\right) d s\right. \\
& \left.\quad-\frac{a_{0}^{k} \tau_{k}^{r_{k}}}{\Gamma\left(r_{k}+1\right)}\right) .
\end{aligned}
$$

We shall prove that $P$ is contractive: Let $\left(u_{1}, u_{2}, \ldots, u_{m}\right),\left(v_{1}, v_{2}, \ldots, v_{m}\right) \in S$. Then, for all $k=1,2, \ldots, m$ and $t \in J$, we get:

$$
\left|P_{k}\left(u_{1}, u_{2}, \ldots, u_{m}\right)(t)-P_{k}\left(v_{1}, v_{2}, \ldots, v_{m}\right)(t)\right|
$$




$$
\begin{aligned}
\leq \frac{1}{\Gamma\left(\alpha_{k}+1\right)} \sup _{s \in J} \sum_{i=1}^{l} \mid f_{i}^{k}\left(s, u_{1}(s), \ldots, u_{m}(s), D^{\gamma_{1}} u_{1}(s), \ldots, D^{\gamma_{m}} u_{m}(s)\right) \\
\quad-f_{i}^{k}\left(s, v_{1}(s), \ldots, v_{m}(s), D^{\gamma_{1}} v_{1}(s), \ldots, D^{\gamma_{m}} v_{m}(s)\right) \mid \\
+\frac{\Gamma\left(r_{k}+n\right) \tau_{k}^{\alpha_{k}+r_{k}}}{(n-1) !\left|\tau_{k}^{r_{k}+n-1}-\Gamma\left(r_{k}+n\right)\right| \Gamma\left(\alpha_{k}+r_{k}+1\right)} \\
\quad \times \sup _{s \in J} \sum_{i=1}^{l} \mid f_{i}^{k}\left(s, u_{1}(s), \ldots, u_{m}(s), D^{\gamma_{1}} u_{1}(s), \ldots, D^{\gamma_{m}} u_{m}(s)\right) \\
\quad-f_{i}^{k}\left(s, v_{1}(s), \ldots, v_{m}(s), D^{\gamma_{1}} v_{1}(s), \ldots, D^{\gamma_{m}} v_{m}(s)\right) \mid .
\end{aligned}
$$

Using $\left(H_{1}\right)$, yields

$$
\begin{aligned}
& \left\|P_{k}\left(u_{1}, u_{2}, \ldots, u_{m}\right)-P_{k}\left(v_{1}, v_{2}, \ldots, v_{m}\right)\right\|_{\infty} \\
& \leq\left(\frac{1}{\Gamma\left(\alpha_{k}+1\right)}+\frac{\Gamma\left(r_{k}+n\right) \tau_{k}^{\alpha_{k}+r_{k}}}{(n-1) !\left|\tau_{k}^{r_{k}+n-1}-\Gamma\left(r_{k}+n\right)\right| \Gamma\left(\alpha_{k}+r_{k}+1\right)}\right) \\
& \quad \times \sum_{i=1}^{l}\left(\left(\mu_{i}^{k}\right)_{1}+\left(\mu_{i}^{k}\right)_{2}+\cdots+\left(\mu_{i}^{k}\right)_{2 m}\right) \max _{1 \leq k \leq m}\left(\left\|u_{k}-v_{k}\right\|_{\infty},\left\|D^{\gamma_{k}}\left(u_{k}-v_{k}\right)\right\|_{\infty}\right) .
\end{aligned}
$$

Thus,

$$
\begin{aligned}
& \left\|P_{k}\left(u_{1}, u_{2}, \ldots, u_{m}\right)-P_{k}\left(v_{1}, v_{2}, \ldots, v_{m}\right)\right\|_{\infty} \\
& \leq \Sigma_{k} \digamma_{k}\left\|\left(u_{1}-v_{1}, \ldots, u_{m}-v_{m}, D^{\gamma_{1}}\left(u_{1}-v_{1}\right), \ldots, D^{\gamma_{m}}\left(u_{m}-v_{m}\right)\right)\right\|_{S} .
\end{aligned}
$$

On the other hand, we have

$$
\begin{aligned}
& \left|D^{\gamma_{k}} P_{k}\left(u_{1}, u_{2}, \ldots, u_{m}\right)(t)-D^{\gamma_{k}} P_{k}\left(v_{1}, v_{2}, \ldots, v_{m}\right)(t)\right| \\
\leq & \frac{t^{\alpha_{k}-\gamma_{k}}}{\Gamma\left(\alpha_{k}-\gamma_{k}+1\right)} \sup _{s \in J} \sum_{i=1}^{l} \mid f_{i}^{k}\left(s, u_{1}(s), \ldots, u_{m}(s), D^{\gamma_{1}} u_{1}(s), \ldots, D^{\gamma_{m}} u_{m}(s)\right) \\
& -f_{i}^{k}\left(s, v_{1}(s), \ldots, v_{m}(s), D^{\gamma_{1}} v_{1}(s), \ldots, D^{\gamma_{m}} v_{m}(s)\right) \mid \\
+ & \frac{\Gamma\left(r_{k}+n\right) t^{n-\gamma_{k}-1} \tau_{k}^{\alpha_{k}+r_{k}}}{\Gamma\left(n-\gamma_{k}\right)\left|\tau_{k}^{r_{k}+n-1}-\Gamma(r+n)\right| \Gamma\left(\alpha_{k}+r_{k}+1\right)} \\
& \times \sup _{s \in J} \sum_{i=1}^{l} \mid f_{i}^{k}\left(s, u_{1}(s), \ldots, u_{m}(s), D^{\gamma_{1}} u_{1}(s), \ldots, D^{\gamma_{m}} u_{m}(s)\right) \\
& \quad-f_{i}^{k}\left(s, v_{1}(s), \ldots, v_{m}(s), D^{\gamma_{1}} v_{1}(s), \ldots, D^{\gamma_{m}} v_{m}(s)\right) \mid .
\end{aligned}
$$

Then,

$$
\begin{aligned}
& \left\|D^{\gamma_{k}} P_{k}\left(u_{1}, u_{2}, \ldots, u_{m}\right)-D^{\gamma_{k}} P_{k}\left(v_{1}, v_{2}, \ldots, v_{m}\right)\right\|_{\infty} \\
\leq & \left(\frac{1}{\Gamma\left(\alpha_{k}-\gamma_{k}+1\right)}+\frac{\Gamma\left(r_{k}+n\right) \tau_{k}^{\alpha_{k}+r_{k}}}{\Gamma\left(n-\gamma_{k}\right)\left|\tau_{k}^{r_{k}+n-1}-\Gamma\left(r_{k}+n\right)\right| \Gamma\left(\alpha_{k}+r_{k}+1\right)}\right)
\end{aligned}
$$




$$
\times \sum_{i=1}^{2 m} \sum_{i=1}^{l} \mu_{i}^{k}\left\|\left(u_{1}-v_{1}, \ldots, u_{m}-v_{m}, D^{\gamma_{1}}\left(u_{1}-v_{1}\right), \ldots, D^{\gamma_{m}}\left(u_{m}-v_{m}\right)\right)\right\|_{S}
$$

So,

$$
\begin{aligned}
& \left\|D^{\gamma_{k}} P_{k}\left(u_{1}, u_{2}, \ldots, u_{m}\right)-D^{\gamma_{k}} P_{k}\left(v_{1}, v_{2}, \ldots, v_{m}\right)\right\|_{\infty} \\
\leq & \Sigma_{k} \digamma_{k}^{*}\left\|\left(u_{1}-v_{1}, \ldots, u_{m}-y_{m}, D^{\gamma_{1}}\left(u_{1}-v_{1}\right), \ldots, D^{\gamma_{m}}\left(u_{m}-v_{m}\right)\right)\right\|_{S} .
\end{aligned}
$$

Thanks to (2.3) and (2.2), we obtain

$$
\begin{aligned}
& \left\|P\left(u_{1}, u_{2}, \ldots, u_{m}\right)-P\left(v_{1}, v_{2}, \ldots, v_{m}\right)\right\|_{S} \\
\leq & \max _{1 \leq k \leq n} \Sigma_{k}\left(\digamma_{k}, \digamma_{k}^{*}\right)\left\|\left(u_{1}-v_{1}, \ldots, u_{m}-v_{m}, D^{\gamma_{1}}\left(u_{1}-v_{1}\right), \ldots, D^{\gamma_{m}}\left(u_{m}-v_{m}\right)\right)\right\|_{S} .
\end{aligned}
$$

By (2.1) the operator $P$ is contractive. Hence, $P$ has a unique fixed point which is a solution of the system (1.1). This ends the proof.

The following result presents new conditions to the existence of one solution. We prove the result by using Schaefer theorem:

Theorem 2.2. Let $\left(f_{i}^{k}\right)_{i=1,2, \ldots, l}^{k=1,2, \ldots, m}$ satisfied $\left(H_{2}\right)$ and $\left(H_{3}\right)$. Then, the problem (1.1) has at least one solution on $J$.

Proof. First, we prove that $P$ is completely continuous. We consider the set

$$
\Omega_{\sigma}:=\left\{\left(u_{1}, u_{2}, \ldots, u_{m}\right) \in S ;\left\|\left(u_{1}, u_{2}, \ldots, u_{m}\right)\right\|_{S} \leq \sigma, \sigma>0\right\},
$$

and we show that $P$ maps bounded sets into bounded sets in $S$.

Let $\left(u_{1}, u_{2}, \ldots, u_{m}\right) \in \Omega_{\sigma}$. The hypothesis $\left(H_{3}\right)$ implies that

$$
\begin{aligned}
& \left\|P_{k}\left(u_{1}, u_{2}, \ldots, u_{m}\right)\right\|_{\infty} \\
\leq & \frac{t^{\alpha_{k}}}{\Gamma\left(\alpha_{k}+1\right)} \sup _{s \in J} \sum_{i=1}^{l}\left|f_{i}^{k}\left(s, u_{1}(s), \ldots, u_{m}(s), D^{\gamma_{1}} u_{1}(s), \ldots, D^{\gamma_{m}} u_{m}(s)\right)\right| \\
& +\left|a_{0}^{k}\right|+\frac{\Gamma\left(r_{k}+n\right) t^{n-1}\left|a_{0}^{k}\right| \tau_{k}^{r_{k}}}{(n-1) !\left|\tau_{k}^{r_{k}+n-1}-\Gamma\left(r_{k}+n\right)\right| \Gamma\left(r_{k}+1\right)} \\
+ & \frac{\Gamma\left(r_{k}+n\right) t^{n-1} \tau_{k}^{\alpha_{k}+r_{k}}}{(n-1) !\left|\tau_{k}^{r_{k}+n-1}-\Gamma\left(r_{k}+n\right)\right| \Gamma\left(\alpha_{k}+r_{k}+1\right)} \\
& \quad \times \sup _{s \in J} \sum_{i=1}^{l}\left|f_{i}^{k}\left(s, u_{1}(s), \ldots, u_{m}(s), D^{\gamma_{1}} u_{1}(s), \ldots, D^{\gamma_{m}} u_{m}(s)\right)\right|
\end{aligned}
$$

$$
\begin{aligned}
\leq & \left(\frac{1}{\Gamma\left(\alpha_{k}+1\right)}+\frac{\Gamma\left(r_{k}+n\right) \tau_{k}^{\alpha_{k}+r_{k}}}{(n-1) !\left|\tau_{k}^{r_{k}+n-1}-\Gamma\left(r_{k}+n\right)\right| \Gamma\left(\alpha_{k}+r_{k}+1\right)}\right) \sup _{s \in J} \sum_{i=1}^{l} \omega_{i}^{k}(s) \\
& +\left|a_{0}^{k}\right|+\frac{\Gamma\left(r_{k}+n\right)\left|a_{0}^{k}\right| \tau_{k}^{r_{k}}}{(n-1) !\left|\tau_{k}^{r_{k}+n-1}-\Gamma\left(r_{k}+n\right)\right| \Gamma\left(r_{k}+1\right)} .
\end{aligned}
$$


Therefore,

$$
\begin{aligned}
& \left\|P_{k}\left(u_{1}, u_{2}, \ldots, u_{m}\right)\right\|_{\infty} \\
\leq & \left(\frac{1}{\Gamma\left(\alpha_{k}+1\right)}+\frac{\Gamma\left(r_{k}+n\right) \tau_{k}^{\alpha_{k}+r_{k}}}{(n-1) !\left|\tau_{k}^{r_{k}+n-1}-\Gamma\left(r_{k}+n\right)\right| \Gamma\left(\alpha_{k}+r_{k}+1\right)}\right) \\
& \times \sum_{i=1}^{l} C_{i}^{k}+\left|a_{0}^{k}\right|+\frac{\Gamma\left(r_{k}+n\right)\left|a_{0}^{k}\right| \tau_{k}^{r_{k}}}{(n-1) !\left|\tau_{k}^{r_{k}+n-1}-\Gamma\left(r_{k}+n\right)\right| \Gamma\left(r_{k}+1\right)} \\
\leq & \digamma_{k} \sum_{i=1}^{l} C_{i}^{k}+W_{k} .
\end{aligned}
$$

Furthermore, we get

$$
\begin{aligned}
& \left\|D^{\gamma_{k}} P_{k}\left(u_{1}, u_{2}, \ldots, u_{m}\right)\right\|_{\infty} \\
\leq & \frac{t^{\alpha_{k}-\gamma_{k}}}{\Gamma\left(\alpha_{k}-\gamma_{k}+1\right)} \sup _{s \in J} \sum_{i=1}^{l}\left|f_{i}^{k}\left(s, u_{1}(s), \ldots, u_{m}(s), D^{\gamma_{1}} u_{1}(s), \ldots, D^{\gamma_{m}} u_{m}(s)\right)\right| \\
& +\frac{\Gamma\left(r_{k}+n\right) t^{n-\gamma_{k}-1}\left|a_{0}^{k}\right| \tau_{k}^{r_{k}}}{\Gamma\left(n-\gamma_{k}\right)\left|\tau_{k}^{r_{k}+n-1}-\Gamma\left(r_{k}+n\right)\right| \Gamma\left(r_{k}+1\right)} \\
& +\frac{\Gamma\left(r_{k}+n\right) t^{n-\gamma_{k}-1} \tau_{k}^{\alpha_{k}+r_{k}}}{\Gamma\left(n-\gamma_{k}\right)\left|\tau_{k}^{r_{k}+n-1}-\Gamma\left(r_{k}+n\right)\right| \Gamma\left(\alpha_{k}+r_{k}+1\right)} \\
& \quad \times \sup _{s \in J} \sum_{i=1}^{l}\left|f_{i}^{k}\left(s, u_{1}(s), \ldots, u_{m}(s), D^{\gamma_{1}} u_{1}(s), \ldots, D^{\gamma_{m}} u_{m}(s)\right)\right| \\
\leq & \left(\frac{1}{\Gamma\left(\alpha_{k}-\gamma_{k}+1\right)}+\frac{\Gamma\left(r_{k}+n\right) \tau_{k}^{\alpha_{k}+r_{k}}}{\Gamma\left(n-\gamma_{k}\right)\left|\tau_{k}^{r_{k}+n-1}-\Gamma\left(r_{k}+n\right)\right| \Gamma\left(\alpha_{k}+r_{k}+1\right)}\right) \\
& \times \sum_{i=1}^{l} \omega_{i}^{k}(t)+\frac{\Gamma\left(r_{k}+n\right)\left|a_{0}^{k}\right| \tau_{k}^{r_{k}}}{\Gamma\left(n-\gamma_{k}\right)\left|\tau_{k}^{r_{k}+n-1}-\Gamma\left(r_{k}+n\right)\right| \Gamma\left(r_{k}+1\right)} .
\end{aligned}
$$

Hence,

$$
\left\|D^{\gamma_{k}} P_{k}\left(u_{1}, u_{2}, \ldots, u_{m}\right)\right\|_{\infty} \leq \digamma_{k}^{*} \sum_{i=1}^{l} C_{i}^{k}+W_{k}^{*} .
$$

From (2.6) and (2.7), we get

$$
\left\|P\left(u_{1}, u_{2}, \ldots, u_{m}\right)\right\|_{S} \leq \max _{1 \leq k \leq m}\left(\digamma_{k} \sum_{i=1}^{l} C_{i}^{k}+W_{k}, \digamma_{k}^{*} \sum_{i=1}^{l} C_{i}^{k}+W_{k}^{*}\right)<\infty .
$$

So, $P$ maps bounded sets into bounded sets in $S$. 
The hypothesis $\left(H_{2}\right)$ implies that $P$ is continuous on $S$. Now, let $t_{1}, t_{2} \in[0,1]$; $t_{1}<t_{2}$, and $\left(u_{1}, u_{2}, \ldots, u_{m}\right) \in S$, we have:

$$
\begin{aligned}
& \left\|P_{k}\left(u_{1}, u_{2}, \ldots, u_{m}\right)\left(t_{2}\right)-P_{k}\left(u_{1}, u_{2}, \ldots, u_{m}\right)\left(t_{1}\right)\right\|_{\infty} \\
\leq & \frac{1}{\Gamma\left(\alpha_{k}+1\right)}\left(2\left(t_{2}-t_{1}\right)^{\alpha_{k}}+\left(t_{2}^{\alpha_{k}}-t_{1}^{\alpha k}\right)\right) \sum_{i=1}^{l} C_{i}^{k} \\
& +\frac{\Gamma\left(r_{k}+n\right)\left|a_{0}^{k}\right| \tau_{k}^{r_{k}}}{(n-1) !\left|\tau_{k}^{r_{k}+n-1}-\Gamma\left(r_{k}+n\right)\right| \Gamma\left(r_{k}+1\right)}\left(t_{2}^{n-1}-t_{1}^{n-1}\right) \\
& +\frac{\Gamma\left(r_{k}+n\right) \tau_{k}^{\alpha_{k}+r_{k}}}{(n-1) !\left|\tau_{k}^{r_{k}+n-1}-\Gamma\left(r_{k}+n\right)\right| \Gamma\left(\alpha_{k}+r_{k}+1\right)}\left(t_{2}^{n-1}-t_{1}^{n-1}\right) \sum_{i=1}^{m} C_{i}^{k},
\end{aligned}
$$

and

$$
\begin{aligned}
& \left\|D^{\gamma_{k}} P_{k}\left(u_{1}, u_{2}, \ldots, u_{m}\right)\left(t_{2}\right)-D^{\gamma_{k}} P_{k}\left(u_{1}, u_{2}, \ldots, u_{m}\right)\left(t_{1}\right)\right\|_{\infty} \\
\leq & \frac{2\left(t_{2}-t_{1}\right)^{\alpha_{k}-\gamma_{k}}+\left(t_{2}^{\alpha_{k}-\gamma_{k}}-t_{1}^{\alpha_{k}-\gamma_{k}}\right)}{\Gamma\left(\alpha_{k}-\gamma_{k}+1\right)} \sum_{i=1}^{l} C_{i}^{k} \\
& +\frac{\Gamma\left(r_{k}+n\right)\left|a_{0}^{k}\right| \tau_{k}^{r_{k}}}{\Gamma\left(n-\gamma_{k}\right)\left|\tau_{k}^{r_{k}+n-1}-\Gamma\left(r_{k}+n\right)\right| \Gamma\left(r_{k}+1\right)}\left(t_{2}^{n-\gamma_{k}-1}-t_{1}^{n-\gamma_{k}-1}\right)
\end{aligned}
$$

$$
+\frac{\Gamma\left(r_{k}+n\right) \tau_{k}^{\alpha_{k}+r_{k}}}{\Gamma\left(n-\gamma_{k}\right)\left|\tau_{k}^{r_{k}+n-1}-\Gamma\left(r_{k}+n\right)\right| \Gamma\left(\alpha_{k}+r_{k}+1\right)}\left(t_{2}^{n-\gamma_{k}-1}-t_{1}^{n-\gamma_{k}-1}\right) \sum_{i=1}^{l} C_{i}^{k} .
$$

The right-hand sides of the above inequalities (2.9) and (2.10) are independent of $\left(u_{1}, u_{2}, \ldots, u_{m}\right)$ and tend to zero as $t_{2}-t_{1} \rightarrow 0$. Then the operator $P$, is equicontinuous. Hence, the operator $P$ is a completely continuous.

Finally we show that

$$
\lambda:=\left\{\left(u_{1}, u_{2}, \ldots, u_{m}\right) \in S, \quad\left(u_{1}, u_{2}, \ldots, u_{m}\right)=\eta P\left(u_{1}, u_{2}, \ldots, u_{m}\right) 0<\eta<1\right\}
$$

is bounded.

For $\left(u_{1}, u_{2}, \ldots, u_{m}\right) \in \lambda$, we have $\left(u_{1}, u_{2}, \ldots, u_{m}\right)(t)=\eta P\left(u_{1}, u_{2}, \ldots, u_{m}\right)(t)$, for all $t \in[0,1]$. Using (2.8), we get

$$
\left\|\left(u_{1}, u_{2}, \ldots, u_{m}\right)\right\|_{S} \leq \eta_{1 \leq k \leq m}\left(\digamma_{k} \sum_{i=1}^{l} C_{i}^{k}+W_{k}, \digamma_{k}^{*} \sum_{i=1}^{l} C_{i}^{k}+W_{k}^{*}\right)<\infty .
$$

Thus, $\lambda$ is a bounded. By Lemma 1.4, the operator $P$ has a fixed point which is a solution of (1.1). Theorem 2.2 is thus proved.

\section{EXAMPLES}

In this section, we give some examples to illustrate our theorems. 
Example 3.1. Consider the following system:

(3.1)

$$
\left\{\begin{aligned}
D^{\frac{11}{3}} u_{1}(t)= & \frac{1}{12 \pi^{3}(t+1)}\left(\cos \left(u_{1}(t)\right)+\cos \left(u_{2}(t)\right)+\frac{\left|D^{\frac{8}{3}} u_{1}(t)+D^{\frac{5}{2}} u_{2}(t)\right|}{\left(1+\left|D^{\frac{4}{3}} u_{1}(t)+D^{\frac{3}{2}} u_{2}(t)\right|\right)}\right) \\
& +\frac{1}{64 \pi(t+1)}\left(\frac{\left|u_{1}(t)+u_{2}(t)\right|}{2 \pi\left(1+\left|u_{1}(t)+u_{2}(t)\right|\right)}+\sin D^{\frac{8}{3}} u_{1}(t)+\sin D^{\frac{5}{2}} u_{2}(t)\right), \\
t \in[0,1], & t \in[0,1], \\
D^{\frac{19}{5}} u_{2}(t)= & \frac{1}{14 \pi^{3} e^{t}}\left(\frac{\left|u_{1}(t)+u_{2}(t)\right|}{1+\left|u_{1}(t)+u_{2}(t)\right|}+\sin \left(D^{\frac{8}{3}} u_{1}(t)\right)+\sin \left(D^{\frac{5}{2}} u_{2}(t)\right)\right) \\
& +\frac{t^{2}}{6 \pi^{3}}\left(\frac{\left|\sin \left(u_{1}(t)\right)+\cos \left(u_{2}(t)\right)+\cos \left(D^{\frac{8}{3}} u_{1}(t)\right)+\sin \left(D^{\frac{5}{2}} u_{2}(t)\right)\right|}{1+\left|\sin \left(u_{1}(t)\right)+\cos \left(u_{2}(t)\right)-\cos \left(D^{\frac{8}{3}} u_{1}(t)\right)+\sin \left(D^{\frac{5}{2}} u_{2}(t)\right)\right|}\right), \\
u_{1}(0)=\sqrt{3}, \quad u_{1}^{(1)}(0)=u_{1}^{(2)}(0)=0, \quad u_{1}^{(3)}(0)=J^{\frac{1}{2}}\left(\frac{3}{7}\right), & u_{2}^{(3)}(0)=J^{\frac{1}{3}}\left(\frac{6}{7}\right) .
\end{aligned}\right.
$$

For this example, we have: $n=4, l=2, \alpha_{1}=11 / 3, \alpha_{2}=19 / 5, \gamma_{1}=8 / 3, \gamma_{2}=5 / 2$, $r_{1}=1 / 2, r_{2}=1 / 3, \tau_{1}=3 / 7, \tau_{2}=6 / 7, J=[0,1]$, and

$$
\begin{aligned}
f_{1}^{1}\left(t, u_{1}, u_{2}, u_{3}, u_{4}\right) & =\frac{1}{12 \pi^{3}(t+1)}\left(\cos u_{1}+\cos u_{2}+\frac{\left|u_{3}+u_{4}\right|}{\left(1+\left|u_{3}+u_{4}\right|\right)}\right), \\
f_{2}^{1}\left(t, u_{1}, u_{2}, u_{3}, u_{4}\right) & =\frac{1}{64 \pi(t+1)}\left(\frac{\left|u_{1}+u_{2}\right|}{2 \pi\left(1+\left|u_{1}+u_{2}\right|\right)}+\sin u_{3}+\sin u_{4}\right), \\
f_{1}^{2}\left(t, u_{1}, u_{2}, u_{3}, u_{4}\right) & =\frac{1}{14 \pi^{3} e^{t}}\left(\frac{\left|u_{1}+u_{2}\right|}{1+\left|u_{1}+u_{2}\right|}+\sin u_{3}+\sin u_{4}\right) \\
f_{2}^{2}\left(t, u_{1}, u_{2}, u_{3}, u_{4}\right) & =\frac{t^{2}}{6 \pi^{3}}\left(\frac{\left|\sin u_{1}+\cos u_{2}+\cos u_{3}+\sin u_{4}\right|}{1+\left|\sin u_{1}+\cos u_{2}-\cos u_{3}+\sin u_{4}\right|}\right) .
\end{aligned}
$$

For all $t \in J$ and $\left(u_{1}, u_{2}, u_{3}, u_{4}\right),\left(v_{1}, v_{2}, v_{3}, v_{4}\right) \in \mathbb{R}^{4}$, we get:

$$
\begin{aligned}
& \left|f_{1}^{1}\left(t, u_{1}, u_{2}, u_{3}, u_{4}\right)-f_{1}^{1}\left(t, v_{1}, v_{2}, v_{3}, v_{4}\right)\right| \\
\leq & \frac{1}{12 \pi^{3}}\left|u_{1}-v_{1}\right|+\frac{1}{12 \pi^{3}}\left|u_{2}-v_{2}\right|+\frac{1}{12 \pi^{3}}\left|u_{3}-v_{3}\right|+\frac{1}{12 \pi^{3}}\left|u_{4}-v_{4}\right| \\
& \left|f_{2}^{1}\left(t, u_{1}, u_{2}, u_{3}, u_{4}\right)-f_{2}^{1}\left(t, v_{1}, v_{2}, v_{3}, v_{4}\right)\right| \\
\leq & \frac{1}{128 \pi^{2}}\left|u_{1}-v_{1}\right|+\frac{1}{128 \pi^{2}}\left|u_{2}-v_{2}\right|+\frac{1}{64 \pi}\left|u_{3}-v_{3}\right|+\frac{1}{64 \pi}\left|u_{4}-v_{4}\right|, \\
& \left|f_{1}^{2}\left(t, u_{1}, u_{2}, u_{3}, u_{4}\right)-f_{1}^{2}\left(t, v_{1}, v_{2}, v_{3}, v_{4}\right)\right| \\
\leq & \frac{1}{14 \pi^{3}}\left|u_{1}-v_{1}\right|+\frac{1}{14 \pi^{3}}\left|u_{2}-v_{2}\right|+\frac{1}{14 \pi^{3}}\left|u_{3}-v_{3}\right|+\frac{1}{14 \pi^{3}}\left|u_{4}-v_{4}\right|
\end{aligned}
$$




$$
\begin{aligned}
& \left|f_{2}^{2}\left(t, u_{1}, u_{2}, u_{3}, u_{4}\right)-f_{2}^{2}\left(t, v_{1}, v_{2}, v_{3}, v_{4}\right)\right| \\
\leq & \frac{1}{6 \pi^{3}}\left|u_{1}-v_{1}\right|+\frac{1}{6 \pi^{3}}\left|u_{2}-v_{2}\right|+\frac{1}{6 \pi^{3}}\left|u_{3}-v_{3}\right|+\frac{1}{6 \pi^{3}}\left|u_{4}-v_{4}\right| .
\end{aligned}
$$

We can take:

$$
\begin{aligned}
& \left(\mu_{1}^{1}\right)_{1}=\left(\mu_{1}^{1}\right)_{2}=\left(\mu_{1}^{1}\right)_{3}=\left(\mu_{1}^{1}\right)_{4}=\frac{1}{12 \pi^{3}} \\
& \left(\mu_{2}^{1}\right)_{1}=\left(\mu_{2}^{1}\right)_{2}=\frac{1}{128 \pi^{2}}, \quad\left(\mu_{2}^{1}\right)_{3}=\left(\mu_{2}^{1}\right)_{4}=\frac{1}{64 \pi}, \\
& \left(\mu_{1}^{2}\right)_{1}=\left(\mu_{1}^{2}\right)_{2}=\left(\mu_{1}^{2}\right)_{3}=\left(\mu_{1}^{2}\right)_{4}=\frac{1}{14 \pi^{3}}, \\
& \left(\mu_{2}^{2}\right)_{1}=\left(\mu_{2}^{2}\right)_{2}=\left(\mu_{2}^{2}\right)_{3}=\left(\mu_{2}^{2}\right)_{4}=\frac{1}{6 \pi^{3}} .
\end{aligned}
$$

Indeed,

$$
\begin{aligned}
& \Sigma_{1} \digamma_{1}=0.001517, \Sigma_{2} \digamma_{2}=0.001820, \\
& \Sigma_{1} \digamma_{1}^{*}=0.022304, \Sigma_{2} \digamma_{2}^{*}=0.027009 .
\end{aligned}
$$

Thus,

$$
\max \left(\Sigma_{1} \digamma_{1}, \Sigma_{2} \digamma_{2}, \Sigma_{1} \digamma_{1}^{*}, \Sigma_{2} \digamma_{2}^{*}\right)<1 .
$$

Using Theorem 2.1, system (3.1) has a unique solution on $J$.

Example 3.2. We consider the following system:

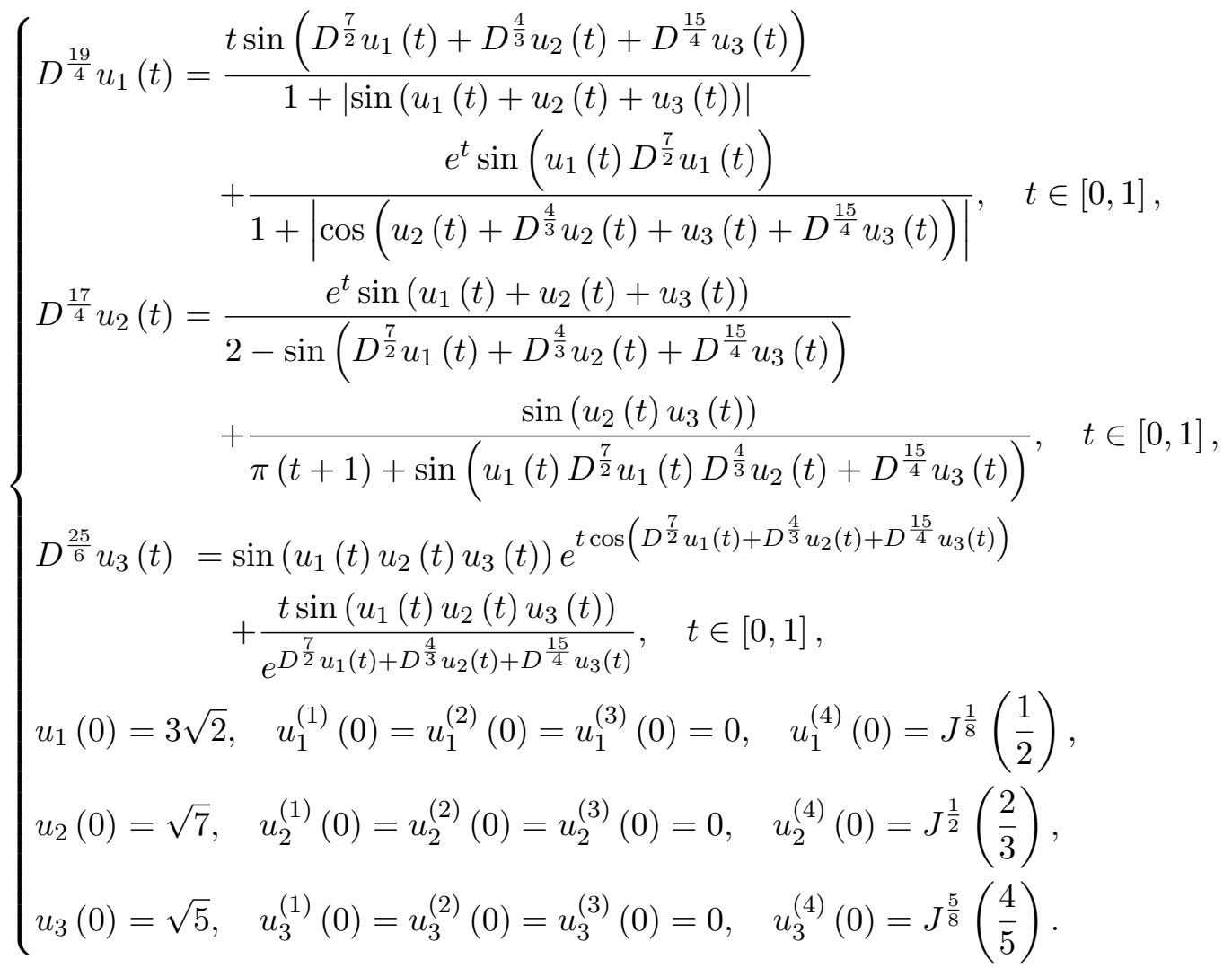


We have: $n=5, l=2, \alpha_{1}=19 / 4, \gamma_{1}=7 / 2, \alpha_{2}=17 / 4, \gamma_{2}=4 / 3, \alpha_{3}=25 / 6$, $\gamma_{3}=15 / 4, r_{1}=1 / 8, r_{2}=1 / 2, r_{3}=5 / 8, \tau_{1}=1 / 2, \tau_{2}=2 / 3, \tau_{3}=4 / 5, J=[0,1]$. And, for all $\left(u_{1}, u_{2}, u_{3}, u_{4}, u_{5}, u_{6}\right) \in \mathbb{R}^{6}$, for all $t \in J$, we get

$$
\begin{aligned}
& \left|f_{1}^{1}\left(t, u_{1}, u_{2}, u_{3}, u_{4}, u_{5}, u_{6}\right)\right| \leq \omega_{1}^{1}(t)=\frac{t\left|\sin u_{4}+u_{5}+u_{6}\right|}{1+\left|\sin \left(u_{1}+u_{2}+u_{3}\right)\right|} \leq C_{1}^{1}=1, \\
& \left|f_{2}^{1}\left(t, u_{1}, u_{2}, u_{3}, u_{4}, u_{5}, u_{6}\right)\right| \leq \omega_{2}^{1}(t)=\frac{e^{t}\left|\sin \left(u_{1} u_{4}\right)\right|}{1+\left|\cos \left(u_{2}+u_{5}+u_{3}+u_{6}\right)\right|} \leq C_{2}^{1}=e, \\
& \left|f_{1}^{2}\left(t, u_{1}, u_{2}, u_{3}, u_{4}, u_{5}, u_{6}\right)\right| \leq \omega_{1}^{2}(t)=\frac{e^{t}\left|\sin \left(u_{1}+u_{2}+u_{3}\right)\right|}{2-\sin \left(u_{4}+u_{5}+u_{6}\right)} \leq C_{1}^{2}=e, \\
& \left|f_{2}^{2}\left(t, u_{1}, u_{2}, u_{3}, u_{4}, u_{5}, u_{6}\right)\right| \leq \omega_{2}^{2}(t)=\frac{\left|\sin \left(u_{2} u_{3}\right)\right|}{\pi(t+1)+\sin \left(u_{1} u_{4} u_{5}+u_{6}\right)} \leq C_{2}^{2}=\frac{1}{\pi-1}, \\
& \left|f_{1}^{3}\left(t, u_{1}, u_{2}, u_{3}, u_{4}, u_{5}, u_{6}\right)\right| \leq \omega_{1}^{3}(t)=\left|\sin \left(u_{1} u_{2} u_{3}\right)\right| e^{t \cos \left(u_{4}+u_{5}+u_{6}\right)} \leq C_{1}^{3}=e, \\
& \left|f_{2}^{3}\left(t, u_{1}, u_{2}, u_{3}, u_{4}, u_{5}, u_{6}\right)\right| \leq \omega_{2}^{3}(t)=\frac{t\left|\sin \left(u_{1}(t) u_{2}(t) u_{3}(t)\right)\right|}{e^{u_{4} t+u_{5}+u_{6}}} \leq C_{2}^{3}=1 .
\end{aligned}
$$

The functions $f_{i}^{k}$ are continuous and bounded on $J \times \mathbb{R}^{6}$. Using Theorem 2.2 , the system (3.2) has at least one solution on $J$.

\section{REFERENCES}

[1] M. A. Abdellaoui and Z. Dahmani, Solvability for nonlinear systems of differential equations with two arbitrary orders, New Zealand J. Math. (to appear).

[2] A. P. Agrawal, M. Benchohra and B. A. Slimani, Existence results for differential equations with fractional order and impulses, Mem. Differ. Equ. Math. Phys. 44 (2008), 1-21.

[3] D. Baleau, S. Z. Nazemi and S. Rezapour, The existence of solution for a $n$ dimentional system of multiterm fractional integrodifferential equations with antiperiodic boundary value problems, Abstr. Appl. Anal. 2014 (2014), Article ID: 896871.

[4] S. Belarbi and Z. Dahmani, Existence of solutions for multi-points fractional evolution equations, Appl. Appl. Math. 9 (2014), 416-427.

[5] Z. Cui, P. Yu and Z. Mao, Existence of solutions for nonlocal boundary value problems of nonlinear differential equations, Adv. Dyn. Syst. Appl. 7 (2012), 31-40.

[6] Z. Dahmani and L. Tabharit, Fractional order differential equations involving caputo derivative, Theory Appl. Math. Comput. Sci. 4 (2014), 40-55.

[7] Z. Dahmani and A. Taieb, New existence and uniqueness results for high dimensional fractional differential systems, Facta Univ. Ser. Math. Inform. 30 (2015), 281-293.

[8] Z. Dahmani and A. Taieb, Solvability of a coupled systems of fractional differential equations with periodic and antiperiodic boundary conditions, Pure and Applied Mathematics Letters 1 (2015), 29-36.

[9] M. Gaber and M. G. Brikaa, Existence results for a coupled system of nonlinear fractional differential equation with three point boundary conditions, J. Fract. Calc. Appl. 21 (2012), 1-10.

[10] R. Hilfer, Application of Fractional Calculus in Physics, World Scientific, Rivers Edge, New Jersey, 2000.

[11] M. Houas and Z. Dahmani, New results for a coupled system of fractional differential equations, Facta Univ. Ser. Math. Inform. 28 (2013), 133-150. 
[12] M. Houas, Z. Dahmani and M. . Benbachir, New results for a boundary value problem for differential equations of arbitrary order, International Journal of Modern Mathematical Sciences 7 (2013), 195-211.

[13] W. H. Jiang, Solvability for a coupled system of fractional differential equations at resonance, Nonlinear Anal. Real World Appl. 13 (2012), 2285-2292.

[14] A. A. Kilbas, H. M. Srivastava and J. J. Trujillo, Theory and Applications of Fractional Differential Equations, vol. 204 of North Holland Mathematics Studies, Elsevier, New York, 2006.

[15] V. Lakshmikantham and A. S. Vatsala, Basic theory of fractional differential equations, Nonlinear Anal. 69 (2008), 2677-2682.

[16] M. Li and Y. Liu, Existence and uniqueness of positive solution for a coupled systems of nonlinear differential equation, Open Journal of Applied Sciences. 3 (2013), 53-61.

[17] F. Mainardi, Fractional calculus: some basic problems in continum and statistical mechanics, in: A. Carpinteri and F. Mainardi (Eds.), Fractals and Fractional Calculus In Continum Mechanics, Springer-Verlag, Wien, 1997, pp. 291-248.

[18] K. S. Miler and B. Ross, An Introduction to the Fractional Calculus and Fractional Differential Equations, Wiley, New York, 1993.

[19] M. D. Ortigueira and J. A. T. Machado, Fractional calculus applications in signals and systems, Signal Prossessing 86 (2006), 2503-2504.

[20] L. Podlubny, Fractional Differential Equations, Academic Press, New York, 1999.

[21] A. Taieb and Z. Dahmani, A coupled systems of nonlinear differential equations involving $m$ nonlinear terms, Georgian Math. J. (2016), DOI: 10.1515/gmj-2016-0014.

[22] C. X. Zhu, X. Zhang and Z. Q. Wu, Solvability for a coupled systems of fractional differential equations with integer boundary conditions, Taiwanese J. Math. 17 (2013), 2039-2054.

${ }^{1}$ Department of Mathematics and Informatics, Faculty SEI, University of Mostaganem,

ALGERIA

E-mail address: lz.tabharit@yahoo.fr

${ }^{2}$ LPAM, FACULTY SEI,

UMAB of Mostaganem,

ALGERIA

E-mail address: zzdahmani@yahoo.fr 